\title{
Prospective evaluation of quality-of-life outcomes following orthotopic neobladder and ileal conduit diversion after radical cystectomy
}

Parier Bastien ${ }^{1}$, Neuzillet Yann ${ }^{2}$, Huyghe Eric ${ }^{3}$, Rouprêt Morgan ${ }^{4}$, Larré Stéphane ${ }^{5}$, Pfister Christian ${ }^{6}$, Mottet Nicolas ${ }^{7}$ and Irani Jacques $^{1 *}$

${ }^{1} \mathrm{CHU}$ Bicêtre Le Kremlin-Bicêtre, France

${ }^{2}$ Hôpital Foch, Suresnes, France

${ }^{3} \mathrm{CHU}$ de Toulouse, Toulouse, France

${ }^{4}$ Hôpital de la Pitié, Paris, France

${ }^{5} \mathrm{CHU}$ de Reims, Reims, France

${ }^{6} \mathrm{CHU}$ de Rouen, Rouen, France

${ }^{7} \mathrm{CHU}$ de Saint-Etienne, Saint-Etienne, France

\begin{abstract}
Objective: The aim of this study was to evaluate health-related quality of life outcomes in patients who underwent orthotopic neobladder reconstruction (ONB) or ileal conduit (IC).

Patients: Eight centers participated in this prospective study that included consecutive patients planned for radical cystectomy. Patients were asked to choose between IC or ONB reconstruction after thorough information. They had to complete the validated self-administered Bladder Cancer Index (BCI) preoperatively and again at 6 and 12 months after surgery as well as a set of questions exploring body image.

Results: Among 106 patients who were invited to participate, 73 completed the preoperative questionnaires. In the preoperative phase, there were no significant differences in any of the QoL domains. At 6 months, 58 (61\%) patients completed their questionnaires showing better functional scores in favor of IC in the urinary domain $(\mathrm{P}=0.01)$. There was no other difference between groups. At 12 months, the findings were similar to those of the 6th month except for body image that was more favorable in ONB patients. VAS assessing diversion overall satisfaction was excellent in both groups despite a poor sexual satisfaction.
\end{abstract}

Conclusions: Even if the results of this prospective non randomized study reflect partly the age difference between groups, they showed a better body image in ONB patients but a better urinary control for IC patients. In both groups, conversely to overall self-assessed satisfaction, sexual satisfaction was poor.

\section{Introduction}

Radical cystectomy is the gold standard management for highrisk urothelial carcinoma of the bladder. It is inevitably followed by urinary diversion (UD) that impacts patients' physical activity, body image and more generally, quality of life (QoL). Thus, the choice of the UD type is an important aspect to consider before surgery. Patients need appropriate counseling in the pre-operative setting although the best form of UD is still a matter of debate and has not been clearly determined [1]. However, the most recent guidelines recommend that orthotopic bladder substitute should be offered to male and female patients who lack any contraindications [2].

The aim of this prospective multicenter study was to evaluate health-related QoL of patients who had ileal conduit (IC) or orthotopic neobladder (ONB) following radical cystectomy for bladder carcinoma.

\section{Material and methods}

Eight French centers participated from 2013 in this prospective non-randomized study that included consecutive patients scheduled to undergo radical cystectomy for urothelial bladder cancer followed by either IC or ONB. Inclusion criteria included a 0-1 WHO performance status score and a 0-1 ASA (American Society of Anaesthesiologists) physical status score. They did not undergo neo-adjuvant chemotherapy and did not have impaired renal function. Patients who agreed to take part were asked to sign a consent form before their surgery. Each patient had to choose between IC and ONB reconstruction, after counseling from his urologist and a specialized nurse.

Patients were asked to complete the validated self-administered Bladder Cancer Index (BCI) before surgery. Postoperatively, at 6 and 12 months, they had to complete again the same questionnaire plus an additional body image questionnaire and a 0 to 10 Visual Analogic Score (VAS) assessing patient's satisfaction regarding their UD.

The BCI used to measure QoL is a validated bladder cancer diseasespecific quality of life instrument. The index consists of 36 items, with

${ }^{*}$ Correspondence to: Jacques Irani, Department of Urology, University Hospital of BICETRE, 94270 Le Kremlin-Bicetre, France, E-mail: jacques.irani@aphp.fr

Key words: bladder cancer, quality of life, ileal conduit, neobladder, radical cystectomy

Received: April 02, 2018; Accepted: April 21, 2018; Published: April 24, 2018 
4- or 5-point Likert response scales, covering three primary domains: urinary (14 items), bowel (10 items), and sexual (12 items). For each domain, a summary score and two subscale scores (function and bother) are constructed. The BCI validation was described in a paper published in 2007 [3] and the French translation was validated three years later [4]. The additional "body image" questionnaire included 10 questions evaluating patient's satisfaction with the appearance of their body and inquiring about any changes that have resulted from the disease and the treatment. Item responses were based on Likert scales with scores from $0-3$ points where lower scores corresponded to better body image $[5,6]$.

ONB techniques were carried out using the techniques published either by Neuzillet et al. [7], Hautmann et al. [8] or Studer et al. [9].

The main outcomes were the comparisons between IC and ONB regarding the BCI three primary domains scores. Secondary outcomes included the comparisons between IC and ONB regarding 1- the BCI subscales, function and bother, for each primary domain 2- the VAS scores and 3- the 10-point body image scale scores.

\section{Results}

One hundred and six patients who met the study criteria accepted to participate. Seventy-three completed the corresponding questionnaires including 44 IC and 29 ONB.

\section{Patients' perioperative characteristics}

Patients' perioperative characteristics are reported in table 1. Patients in the IC group were older and had a more advanced. Baseline urinary, bowel and sexual health were not different between the IC and the ONB patients. Mean length of hospitalization was 19 days (range 7-51) with no significant differences between groups.

At 6 months, 58 patients completed their questionnaires (30 IC and $18 \mathrm{ONB})$. BCI questionnaires analyzed item by item showed significant differences in favor of the ONB group regarding constipation $(p=0.03)$ and urinary problems/complaints, mostly because of stoma problems $(\mathrm{p}=0.03)$. On the other hand, the IC group reported better urinary control and less leakage compared to the ONB group $(\mathrm{p}=0.02)$. There was no significant difference in BCI scale scores between the IC and ONB groups: median scores for urinary health were 83 vs 72 respectively ( $\mathrm{p}=0.1)$, for bowel health, 85 vs $83(\mathrm{p}=0.5)$ and for sexual health, 38 vs 33 ( $\mathrm{p}=0.1)$ (Figure 1$)$.

When considering the two subdomains (function and bother), the function subscale of the urinary domain was in favor of IC compared to ONB ( 83 vs $42 ; \mathrm{p}=0.0005$ ). There was no difference between UD groups for any of the other subdomains (Figure 2). Results of the body image questionnaire were not significantly different at 6 month (Figure 3). VAS assessing UD overall satisfaction was excellent in both groups without significant difference (table 2). Results were not modified when performing a multivariate analysis taking into account age and stage.

Table 1. Peri-operative characteristics by diversion procedure

\begin{tabular}{|c|c|c|c|}
\hline & Ileal conduit & Orthotopic neobladder & p \\
\hline No. of patients, $\mathrm{n}(\%)$ & $44(62 \%)$ & $29(38 \%)$ & - \\
\hline Mean age (SD), years & $69.9(9.4)$ & $64.4(6.8)$ & $\mathbf{0 . 0 0 2}$ \\
\hline Sex, n (\%) & & & \\
Male & $34(77.3 \%)$ & $24(82.8 \%)$ & 0.2 \\
Female & $10(22.7 \%)$ & $5(17.2 \%)$ & 0.2 \\
\hline Stage $>=$ pT3 & $57.6 \%$ & $29.2 \%$ & $\mathbf{0 . 0 3}$ \\
\hline Stage pN+ & $31.2 \%$ & $32.0 \%$ & 0.3 \\
\hline
\end{tabular}

Table 2. Visual Analogic scale (VAS) from 0 to $10(0=$ not satisfied at all and $10=$ very satisfied) evaluating patient's satisfaction with his urinary diversion at 6 and 12 months. Results are expressed as median value (IQ)

\begin{tabular}{|c|c|c|c|}
\hline & IC & ONB & $\boldsymbol{p}$ \\
\hline VAS at 6 months & $9.0(1.6)$ & $10.0(1.5)$ & 0.2 \\
\hline VAS at 12 months & $9.0(1.6)$ & $9.7(1.0)$ & 0.2 \\
\hline
\end{tabular}

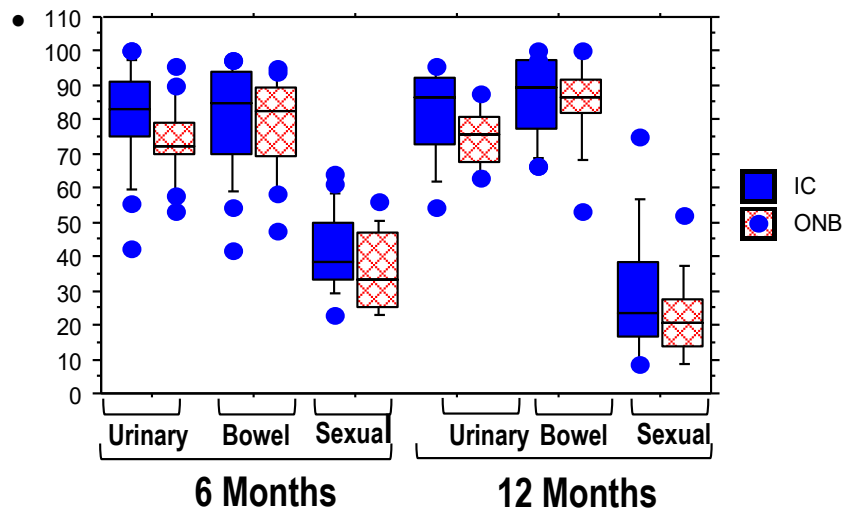

Figure 1. Box plots composed of five lines displaying the 10th, 25th, 50th, 75th and 90th percentiles of Bladder Cancer Index (BCI) primary domains scores measuring urinary, bowel, and sexual health. Blue dots indicate values falling outside the $10^{\text {th }}$ or $90^{\text {th }}$ percentile. Item responses are based on Likert scales standardized to a $0-100$ point scale where higher scores correspond to better health states. There was no significant difference between IC and ONB scores.

At 12 months, 34 patients completed their questionnaires (19 IC; 15 ONB). Body image scores were significantly more favorable in the ONB group (median scores 7 vs 2.5 for IC and ONB respectively; $\mathrm{p}=0.03$ ) (Figure 3 ). The findings were similar to those of the $6^{\text {th }}$ month regarding BCI domains (Figure 1). IC patients performed also significantly better than ONB patients at 12 months for the function subscale of the urinary domain ( 83 vs $58 ; \mathrm{p}=0.009$ ) (figure 2). VAS assessing UD overall satisfaction remained excellent in both groups (table 2). Results were not modified when performing a multivariate analysis taking into account age and stage.

\section{Complications and recurrence}

Within the first year following surgery, 15 patients presented complications requiring rehospitalization for medical treatment and/ or surgical, endoscopic or radiological intervention. There was no significant difference between groups. Five patients were diagnosed with cancer recurrence, four in the IC group and one in the ONB group. No patient died.

\section{Adjuvant chemotherapy}

There was no significant difference between patients who had or did not have chemotherapy when comparing BCI, body image and VAS scores.

\section{Discussion}

IC has been considered for decades the "standard" urinary diversion for bladder cancer patients submitted to radical cystectomy and remains in most countries the most commonly used diversion after radical cystectomy [10]. During the last two decades, this surgical procedure has been challenged by the dissemination and the good clinical outcome of bladder substitutions. However, a debate is still ongoing due to a lack of solid evidence. Our study did not find significant differences between the two types of diversions except for functional urinary health and body image, in favor of IC for the former 

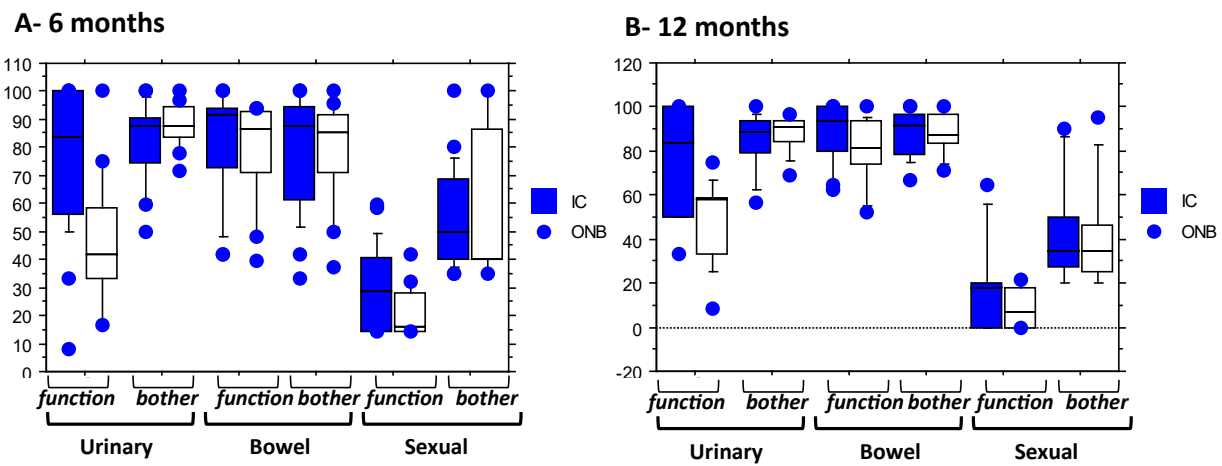

Figure 2A and 2B. Box plots composed of five lines displaying the 10th, 25th, 50th, 75th and 90th percentiles of Bladder Cancer Index (BCI) subdomains -function and bother- scores for each primary domain. Blue dots indicate values falling outside the $10^{\text {th }}$ or $90^{\text {th }}$ percentile. Item responses are based on Likert scales, with subdomain scores standardized to a $0-100$ point scale where higher scores correspond to better health states.

Figure 2A. At 6 months, a significant difference was seen only for the function subscale of the urinary domain ( 83 vs 42 ; $\mathrm{p}=0.0005)$, while there was no difference between the diversion groups for any of the other subdomains.

Figure 2B. At 12 months, IC group patients performed also significantly better than the ONB patients for the function subscale of the urinary domain ( 83 vs 58 ; $\mathrm{p}=0.009$ ), while there was no difference between groups for the other subdomains.

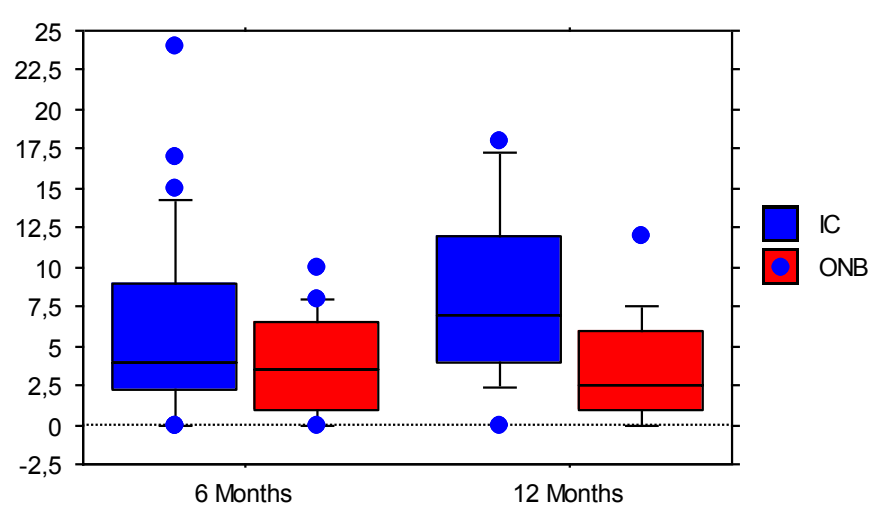

Figure 3. Box plots composed of five lines displaying the 10th, 25th, 50th, 75th and 90th percentiles of Bladder Cancer Index (BCI) body image 10-question questionnaire scores at 6 and 12 months. Blue dots indicate values falling outside the $10^{\text {th }}$ or $90^{\text {th }}$ percentile. Item responses are based on Likert scales with scores from $0-3$ points where lower scores correspond to better body image. Results were significantly less favorable at 12 months in the IC group compared to the ONB group: median value (IQ) $=7$ (8) and 2.5 (5) respectively $(\mathrm{p}=0.03)$.

item and of ONB for the latter. When analyzing the different items of the urinary domain, the ONB group more commonly reported frequent urinary leakage and occasional urinary dribbling compared with the IC group. Regarding body image results, it should be noted that a higher proportion of IC patients were lost to follow-up which may constitutes a bias influencing the results.

It could appear initially to most patients and even practitioners that ONB should be the standard diversion following radical. However, clinical experience shows that ONB has functional limitations [11] and that its rate of complications in the long term is substantial and possibly higher than that of IC $[12,13]$. On the other hand, a pouching system can be in some patients significantly detrimental to their body image with secondary consequences on various daily activities. There are no randomized studies - which are non-feasible - and patients cannot be their own controls as they will never experience the other UD. Secondly, appraising the impact of a specific UD on QoL and moreover comparing the impact of different UDs is a complex issue where various baseline characteristics including age, disease stage and socio-cultural aspects may constitute a substantial source of heterogeneity. As for the impact of UD evaluation, we decided to use a validated, self-administered and specific questionnaire. Although the
BCI questionnaire is more specific in the context of this study than a generic instrument, it is still subject to limitations, one being the gender neutral questions that cannot identify issues comparing men and women particularly for the sexual domain. Additionally, we added a VAS to assess patient's overall satisfaction and a 10-item body-image questionnaire $[5,6]$.

This study has several limitations. Questionnaire response rates reduced over time despite reminders. This is a potential source of bias as we could not gather information about the non-responders group beyond age and gender. Selection bias related to the non-randomized design of this study was clearly reflected by the finding that average age was higher in those patients choosing to undergo IC for their diversion, although this reflects real-world clinical practice.

Several studies aimed to evaluate QoL following different UD. Common limitations included non-validated health related quality of life (HRQOL) instruments, use of general HRQOL instruments only, lack of baseline data, cross- sectional analysis, and retrospective study design [14]. Most of these studies concluded that there was no evidence that one form of urinary diversion was superior to another based on HRQOL outcomes [14-17]. Few studies on the other hand reported marginal quality of life advantages with ONB [18-20] or conversely showed a better QoL for IC [6,21]. Several hypotheses could explain the outcome differences between the studies such as different populations, different study design (retrospective or prospective cohorts), different follow-ups, but also different means of evaluating QoL. We have recently published a systematic review on IC and ONB comparison and the main finding was that IC patients demonstrated a better HR-QoL in urinary outcomes than ONB patients [22].

\section{Conclusion}

Even if the results of this study reflect partly the age difference between groups, they showed an advantage for the neobladder diversion regarding body image but an advantage for the ileal conduit diversion regarding functional urinary health. Beside these items, no difference was demonstrated for bowel and sexual domains as well as overall self-assessed diversion satisfaction. The type of diversion should follow a shared decision between an informed patient and health staff and ileal conduit should not be reduced to the rank of an alternative in case of ONB contra-indication. 


\section{References}

1. Stenzl A, Cowan NC, De Santis M, Kuczyk MA, Merseburger AS, et al. (2011) Treatment of muscle-invasive and metastatic bladder cancer: update of the EAU guidelines. Eur Urol 59: 1009-1018. [Crossref]

2. Witjes JA, Compérat E, Cowan NC, De Santis M, Gakis G, et al. (2014) EAU guidelines on muscle-invasive and metastatic bladder cancer: summary of the 2013 guidelines. Eur Urol 65: 778-792. [Crossref]

3. Gilbert SM, Wood DP, Dunn RL, Weizer AZ, Lee CT, et al. (2007) Measuring healthrelated quality of life outcomes in bladder cancer patients using the Bladder Cancer Index (BCI). Cancer 109: 1756-1762. [Crossref]

4. Gaunez N, Larré S, Pirès C, Doré B, Wei J, et al. (2010) [French translation and linguistic validation of the questionnaire Bladder Cancer Index (BCI)]. Prog Urol 22: 350-353. [Crossref]

5. Hopwood P, Fletcher I, Lee A, Al Ghazal S (2001) A body image scale for use with cancer patients. Eur J Cancer 7: 189-197. [Crossref]

6. Hedgepeth RC, Gilbert SM, He C, Lee CT, Wood DP Jr (2010) Body image and bladder cancer specific quality of life in patients with ileal conduit and neobladder urinary diversions. Urology 76: 671-675. [Crossref]

7. Neuzillet Y, Yonneau L, Lebret T, Herve JM, Butreau M, et al. (2011) The Z-shaped ileal neobladder after radical cystectomy: an 18 years' experience with 329 patients. BJU Int 108: 596-602. [Crossref]

8. Hautmann RE, Egghart G, Frohneberg D, Miller K (1988) The ileal neobladder. $J$ Urol 139: 39-42. [Crossref]

9. Studer UE, Danuser H, Hochreiter W, Springer JP, Turner WH, et al. (1996) Summary of 10 years' experience with an ileal low pressure bladder substitute combined with an afferent tubular peristaltic segment. World J Urol 14: 29-39. [Crossref]

10. Gore JL, Litwin MS; Urologic Diseases in America Project (2009) Quality of care in bladder cancer: trends in urinary diversion following radical cystectomy. World $J$ Urol 27: 45-50. [Crossref]

11. Ahmadi H, Skinner EC, Simma-Chiang V, Miranda G, Cai J, et al. (2013) Urinary functional outcome following radical cystoprostatectomy and ileal neobladder reconstruction in male patients. J Urol 189: 1782-1788. [Crossref]
12. Nieuwenhuijzen JA, de Vries RR, Bex A, van der Poel HG, Meinhardt W, et al. (2008) Urinary diversions after cystectomy: the association of clinical factors, complications and functional results of four different diversions. Eur Urol 53: 834-844. [Crossref]

13. Siddiqui K, Izawa1 J (2016): Ileal conduit: standard urinary diversion for elderly patients undergoing radical cystectomy. World J Urol 34: 19-24. [Crossref]

14. Porter MP, Penson DF (2005) Health related quality of life after radical cystectomy and urinary diversion for bladder cancer: a systematic review and critical analysis of the literature. J Urol 173: 1318-1322. [Crossref]

15. Somani BK, Nabi G, Wong S, Lyttle M, Atiemo K, et al. (2009) How close are we to knowing whether orthotopic bladder replacement surgery is the new gold standard?evidence from a systematic review update. Urology 74: 1331-1339. [Crossref]

16. Large MC, Malik R, Cohn JA, Richards KA, Ganshert C, et al. (2014) Prospective Health-related Quality of Life Analysis for Patients Undergoing Radical Cystectomy and Urinary Diversion. Urology 84: 808-813. [Crossref]

17. Ali AS, Hayes MC, Birch B, Dudderidge T, Somani BK (2015) Health related quality of life (HRQoL) after cystectomy: comparison between orthotopic neobladder and ileal conduit diversion. Eur J Surg Oncol 41: 295-299. [Crossref]

18. Hobisch A, Tosun K, Kinzl J, Kemmler G, Bartsch G, et al. (2000) Quality of life after cystectomy and orthotopic neobladder versus ileal conduit urinary diversion. World $J$ Urol 18: 338-344. [Crossref]

19. Dutta SC, Chang SC, Coffey CS, Smith JA Jr, Jack G, et al. (2002) Health related quality of life assessment after radical cystectomy: comparison of ileal conduit with continent orthotopic neobladder. J Urol 168: 164-167. [Crossref]

20. Singh V, Yadav R, Sinha RJ, Gupta DK (2014) Prospective comparison of qualityof-life outcomes between ileal conduit urinary diversion and orthotopic neobladder reconstruction after radical cystectomy: a statistical model. BJU Int 113: 726-732. [Crossref]

21. Goldberg H, Baniel J, Mano R, Rotlevy G, Kedar D, et al. (2016) Orthotopic neobladde vs. ileal conduit urinary diversion: A long-term quality-of-life comparison Urologic Oncology: Seminars and Original Investigations 34: 121.e1-121.e7.

22. Ziouziou I, Irani J, Wei JT, Karmouni T, El Khader K, et al. (2018) Ileal conduit vs orthotopic neobladder: Which one offers the best health-related quality of life in patients undergoing radical cystectomy? A systematic review of literature and metaanalysis. Prog Urol 28: 241-250. [Crossref]

Copyright: (C2018 Bastien P. This is an open-access article distributed under the terms of the Creative Commons Attribution License, which permits unrestricted use, distribution, and reproduction in any medium, provided the original author and source are credited. 